\title{
Benefícios e soluções da integração de BIM-FM e gestão de espaços
}

Benefits and solutions for the integration of BIM-FM and space management

\author{
Alessandra Teixeira \\ Universidade Federal do Paraná | Curitiba | Brasil | alessandrateixeira.t@gmail.com \\ Sergio Scheer \\ Universidade Federal do Paraná | Curitiba | Brasil | sergioscheer@gmail.com
}

\section{Resumo}

A fase operacional do ciclo de vida de imóveis apresenta relevância para proprietários em razão de sua maior duração. Gestão de espaços é um segmento de Gerenciamento de Instalações e sua integração com a Modelagem da Informação da Construção pode gerar benefícios para gestores. O objetivo deste estudo é coletar e classificar, por meio de um Revisão Sistemática da Literatura, pesquisas que abordam gestão de espaços a partir de aplicações BIM-FM. Os resultados constataram que há lacunas nas definições existentes de gestão de espaços visto a difusão de novas tecnologias e a atenção para a experiência do usuário nos espaços.

Palavras-chave: Modelagem da Informação da Construção. Gerenciamento de Instalações. Gestão de espaços. Revisão Sistemática da Literatura.

\begin{abstract}
The operational phase of a facility llifecycle is relevance to owners because of longest duration. Space management is a facility management segment and the integration with Building Information Modeling can generate benefits for managers. The aims of this research is to collect and classify, through a Literature Systematic Review, studies that addresses space management from BIM-FM applications. The results detect that there are gaps in the existing definitions of space management due to the diffusion of new technologies and attention to the user experience in the spaces.
\end{abstract}

Keywords: BIM. Facility Management. Space management. Literature Systematic Review.

\section{INTRODUÇÃO}

A gestão eficiente de ativos imobiliários apresenta relevância para proprietários e organizações, de modo que a etapa de manutenção e operação dos imóveis possui a maior duração em comparação com as demais fases de seu ciclo de vida.

Na administração pública o princípio da economicidade se destaca, uma vez que parte dos recursos aplicados na gestão dos imóveis advém do pagamento de impostos pela população.

Como citar:

TEIXEIRA , A.; SCHEER , S. Benefícios e soluções da integração de BIM-FM e gestão de espaços. In: SIMPÓSIO BRASILEIRO DE TECNOLOGIA DA INFORMAÇÃO E COMUNICAÇÃO NA CONSTRUÇÃO, 3., 2021, Uberlândia. Anais [...]. Porto Alegre: ANTAC, 2021. p. 1-10. Disponível em:

https://eventos.antac.org.br/index.php/sbtic/article/view/579. Acesso em: 3 ago. 2021. 
Neste cenário, as tecnologias da informação e comunicação (TICS) são inseridas como o centro de mudanças de impacto para governos, empresas e no bem-estar dos cidadãos [1].

A Modelagem da Informação da Construção (Building Information Modeling - BIM) é uma das principais alternativas para melhorar o desempenho da indústria de Arquitetura, Engenharia e Construção e Operação (AECO), de modo que para a fase de operação do imóvel, caracteriza-se o uso de BIM integrado ao gerenciamento de instalações (Facility Management - FM), denominado BIM-FM [2].

O gerenciamento de instalações tem como segmento a "gestão de bens imóveis ou locais que fornecem espaço para organizações" [3], no qual BIM pode ser implementado e trazer diversos benefícios.

Desta forma, este estudo tem como objetivo coletar, interpretar e classificar pesquisas científicas que abordam a gestão de espaços a partir de aplicações BIM-FM a fim de compreender quais são as soluções identificadas e benefícios de seu uso na gestão e operação de imóveis.

\section{MÉTODO}

Revisões sistemáticas da literatura (RSL) são estudos em que se utiliza de um método explícito, planejado e executado com rigor para agregar pesquisas primárias relevantes acerca de um tópico de pesquisa, de maneira que ao consolidar e agrupar as pesquisas torna-se possível encontrar um novo conhecimento [4].

Para o presente estudo adotou-se o procedimento de uma RSL, no qual as fontes de busca utilizadas foram bases de dados eletrônicas Web of Science e Scopus/ Elsevier, com a inclusão de pesquisas relevantes que não apareceram na busca inicial. Os termos utilizados foram "BIM", "FM ou Facility / Facilities" e "space (espaço)", junto com operador booleano AND que limitam a busca as pesquisas que contenham todas as palavras listadas independentemente de sua ordem. Os termos foram buscados em títulos, resumos e palavras-chave.

Devido à abrangência dos termos utilizados na busca, como critério de exclusão se optou por eliminar da amostra as pesquisas que tratam de gestão de espaço em canteiros de obras, técnicas de documentação (as-built) como laser scanner ou nuvem de pontos e estudos que tratam de empreendimentos de infraestrutura.

Foram encontrados um total de 61 artigos e anais de eventos publicados no recorte temporal do ano 2000 até agosto de 2020, conforme síntese do Quadro 1. 
Quadro 1: Protocolo de condução da RSL

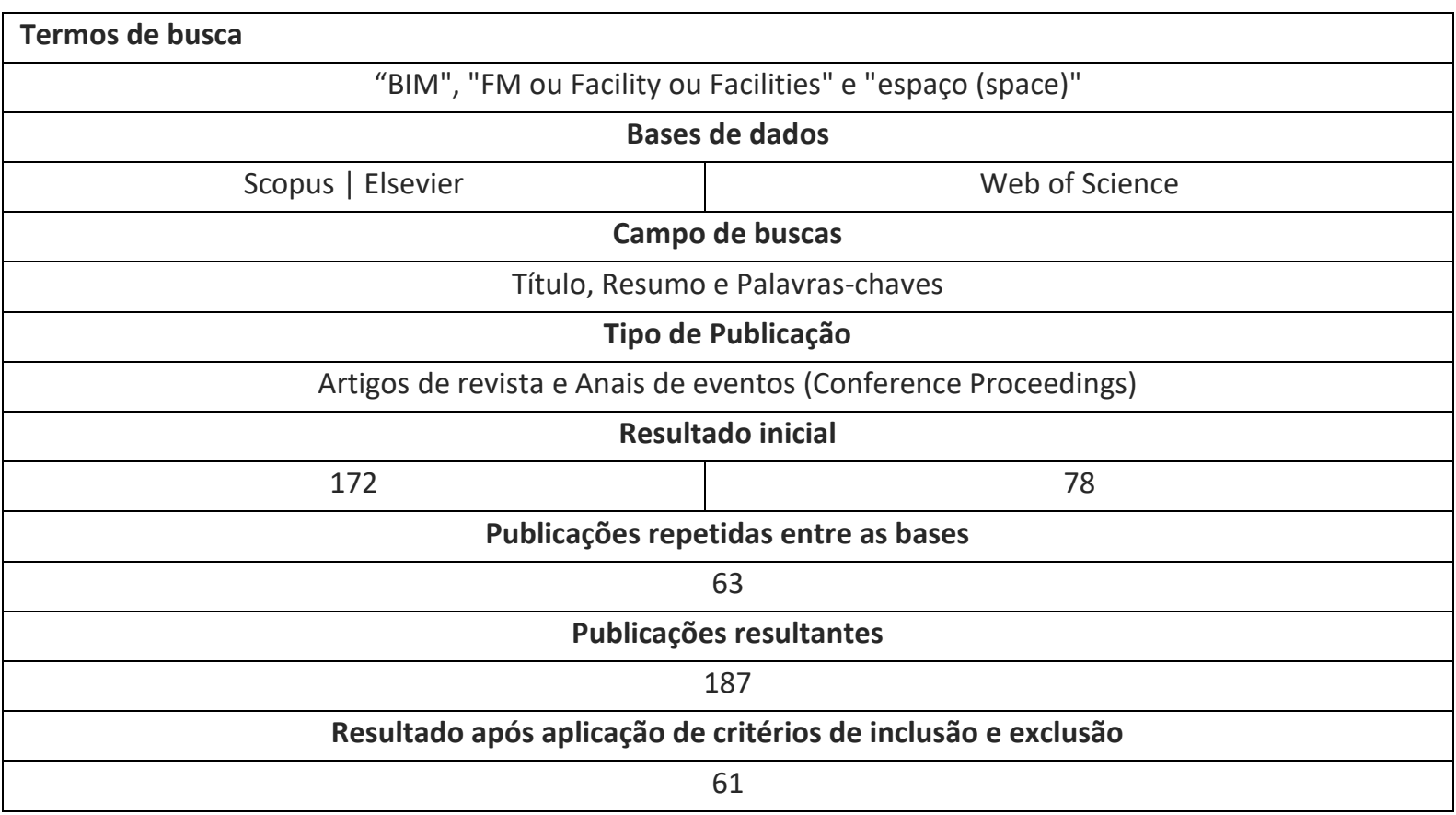

Fonte: os autores.

\section{RESULTADOS}

Verificou-se que a busca inicial abrangeu 187 pesquisas não repetidas, das quais foram limitadas ao recorte do presente estudo, resultando em 61 publicações. Em razão da escolha de não utilizar o termo gestão de espaço ou space management, foi observado por meio de uma leitura inspecional que diversas pesquisas não tratavam de gestão de espaços como objetivo principal, no entanto abordavam o assunto de forma secundária, citando ou utilizando-o como meio para atingir os objetivos propostos. Desta forma, em uma segunda etapa a amostra foi classificada por eixos temáticos que correspondem a alguns dos reconhecidos usos de BIM-FM [5] esquematizados conforme Figura 1.

Figura 1: Classificação da amostra conforme eixos temáticos

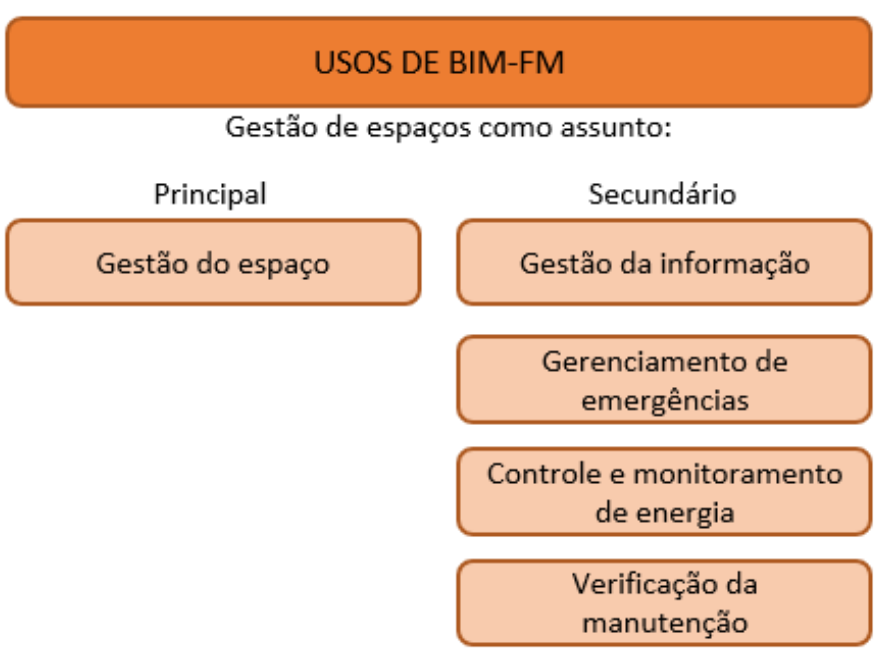

Fonte: os autores. 
O quantitativo de pesquisas de acordo com cada eixo temático pode ser verificado no Gráfico 1.

Figura 2: Número de pesquisa por eixo temático

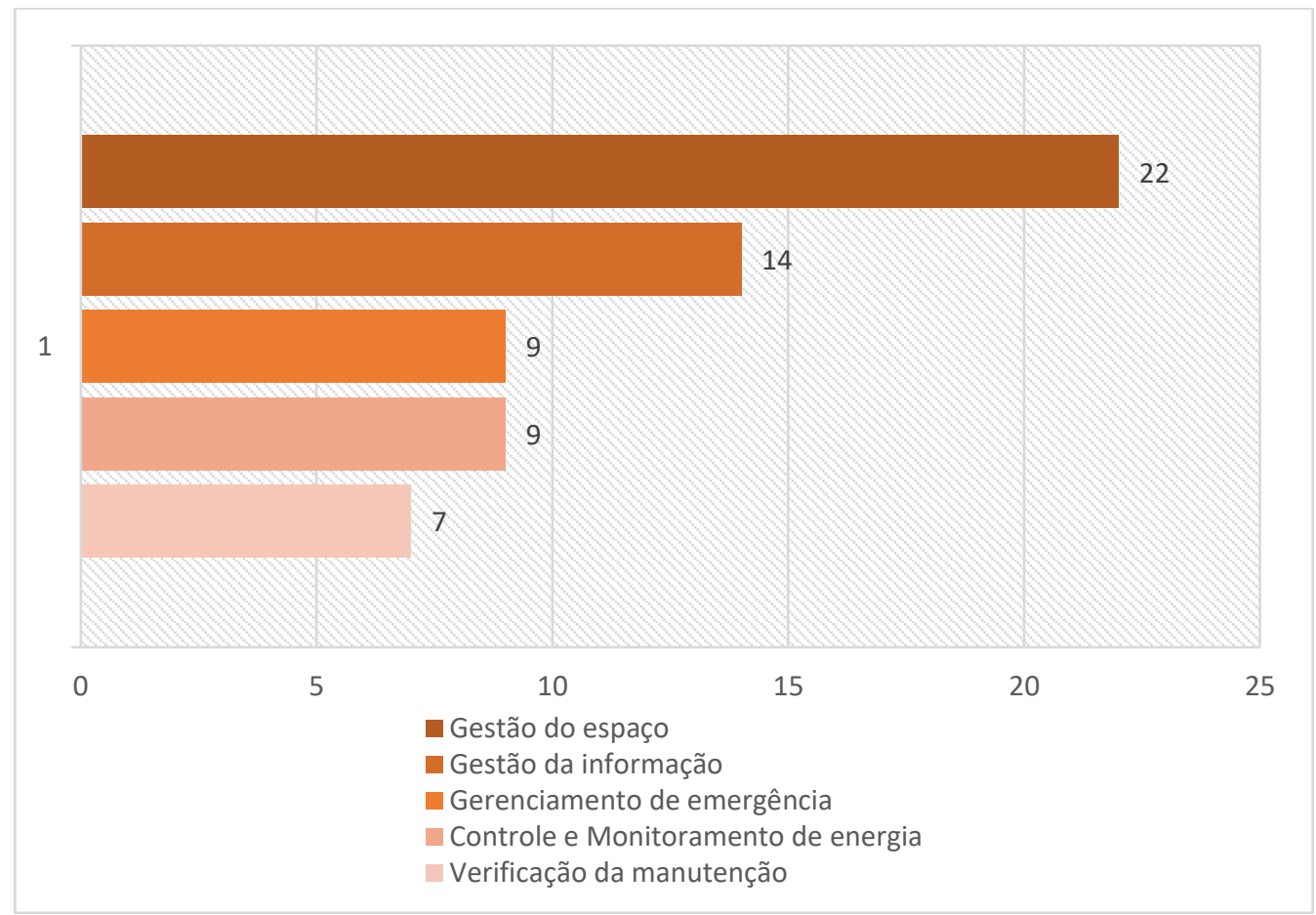

Fonte: os autores.

Realizou-se a leitura analítica das 22 pesquisas em que a gestão de espaços foi identificada como assunto principal e foi realizado um agrupamento de acordo com classes de problemas observadas nas quais buscam-se melhorias a partir de soluções integradas à modelos BIM-FM. A classificação realizada abrangeu o conforto interno de espaços, ocupação e alocação de espaços, inventário de espaços, simulações de usos de espaços e visualização de espaços pelo usuário. As referências da bibliografia de acordo com a classificação desenvolvida constam no Quadro 2.

Quadro 2: Classes de problemas abordadas com gestão de espaços em BIM-FM

\begin{tabular}{|l|l|}
\hline Classificação & Referências \\
\hline Conforto interno de espaços & $6 ; 7 ; 8 ; 9 ; 10 ; 11 ;$ \\
\hline Ocupação/ alocação de espaços & $6 ; 12 ; 13 ; 14 ; 15 ;$ \\
\hline Inventário de espaços & $6 ; 10 ; 11 ; 14 ; 15 ; 16 ; 17 ; 18 ; 19 ; 20 ;$ \\
\hline Simulações de usos de espaços & $21 ; 22 ; 23 ;$ \\
\hline Visualização de espaços pelo usuário & $23 ; 24 ; 25 ; 26 ; 27 ;$ \\
\hline
\end{tabular}

Fonte: os autores.

A partir do agrupamento foi possível caracterizar conforme os itens subsequentes as abordagens dos autores em relação a cada classe de problema, benefícios e melhorias promovidos em suas pesquisas. 
Verificam-se benefícios para o gestor e proprietário do imóvel, assim como para os usuários que recebem serviços de melhor qualidade, melhores condições internas dos espaços e de forma mais rápida [10]. As visualizações de chamados de reclamações quanto ao conforto térmico, qualidade do ar e acústica dos espaços podem ser destacadas em modelos BIM-FM e ainda, os feedbacks de usuários podem ser visualizados no local ou espaço em que o problema foi observado [6][7][9]. Constatase uso de sensores, dispositivos de Internet das Coisas (IoT) para coletar e atualizar dados das condições internas dos espaços [8] e gêmeos digitais (Digital Twins - DT) que podem representar em sua plataforma as condições internas em tempo real, modelo as-is [11].

\section{OCUPAÇÃO/ ALOCAÇÃO DE ESPAÇOS}

Os softwares de FM disponíveis comercialmente, que integram modelos BIM, possibilitam diversas funções relacionadas as alocações de espaços como a comparação de espaços necessários com os espaços disponíveis, reserva de espaços, o controle de informações de pessoal e equipamentos associados aos espaços, identificação de espaços ocioso, gráficos de vacância e o planejamento da ocupação [6][13][14]. Ainda, sensores podem ser utilizados para monitoramento da ocupação que geram informações sobre a real utilização do espaço e podem contribuir para gerentes de instalações no planejamento de ações de manutenção [15].

\section{INVENTÁRIO DE ESPAÇOS}

Destaca-se o valor agregado para gerentes e proprietários ao disponibilizar dados visuais dos espaços por meio de modelos BIM-FM para referências e análises de maneira ágil como suporte para tomadas de decisões [6]. Possibilitam um melhor entendimento das edificações existentes, a semântica dos espaços e relações com espaços adjacentes [16]. Os inventários de informações dos espaços podem ser utilizados navegação interna por usuários [17], detectar distâncias e rotas entre espaços [16], definir limites de áreas privativas e comuns em edifícios que possuem diferentes proprietários [19] e limites da abrangência de dispositivos loT [20].

\section{SIMULAÇÕES DE USOS DOS ESPAÇOS}

Simulações de circulação de usuários podem ser realizadas a partir de algoritmos que relacionam as rotas de circulação com as propriedades dos elementos de espaços como portas, escadas e elevadores [21]. A partir do registro de atividades de usuários no imóvel são desenvolvidas simulações de circulações de pessoas, análise de áreas com acúmulo de tráfego de pessoas e proposições de melhorias que incluem a proposição de alterações de espaços [22]. A utilização de realidade virtual (VR) para simulações de aglomerações de pessoas proporciona a percepção de usuário ao gerente e pode gerar reavaliações do uso atual dos espaços [23]. 
Verifica-se a preocupação com a experiência do usuário no imóvel [23]. O uso de realidade virtual em celulares aliada a modelos BIM permite a visualização de informações no local correspondente de utilização do celular [24]. O uso de BIM e Sistemas de Informações Geográficas - SIGs em plataformas WEB pode ser útil para usuários de Campus de Universidades para navegar pelos imóveis e traçar rotas de deslocamento [26]. As simulações computacionais podem ajudar no desenvolvimento de cenários com complexidades diferentes, de modo que gerentes, funcionários e usuários dos serviços podem analisar as interações das pessoas e dos espaços físicos [27].

Em atenção ao contexto das pesquisas foram elencados os cenários de aplicação dos trabalhos que tratam a gestão de espaço como assunto principal conforme Gráfico 2. Observa-se que a maior parcela das pesquisas utilizou universidades e escolas como objeto de análise.

Figura 3: Cenários de aplicação das pesquisas selecionadas

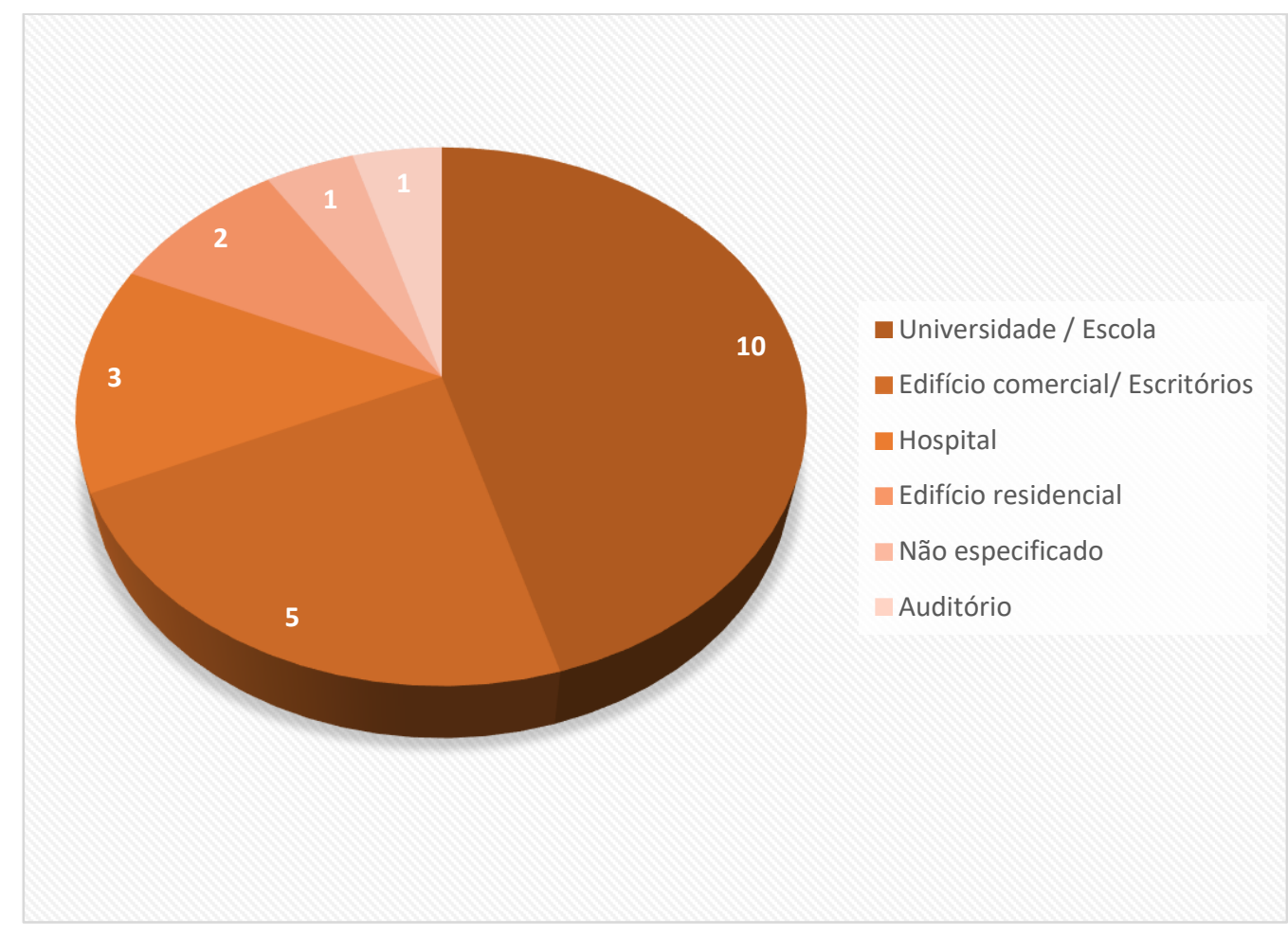

Fonte: os autores.

\section{DISCUSSÃO}

Diante do exposto foi possível identificar, classificar e sintetizar os problemas na gestão de espaços nos quais as aplicações BIM-FM apresentam benefícios e melhorias.

Em razão da abrangência dos termos de buscas utilizados, por meio do Gráfico 1 se evidencia que dentre a amostra de 61 pesquisas, a parcela de 39 tratam de usos de BIM-FM que fazem referência a gestão de espaços, mas não como tema principal. 
Revela-se uma importância do assunto, visto sua presença também como intermediário para os demais usos de BIM-FM.

A estratificação realizada, a qual identificou as classes de problemas, possibilitou a comparação das aplicações observadas com as descrições de gestão de espaços em BIM-FM de publicações tidas como referências devido à grande difusão na academia e indústria.

A definição de gestão de espaços como "um uso do modelo, onde modelos 3D são usados para gerenciar a ocupação de salas e espaços dentro de ativos físicos" [28] apresenta-se como uma descrição sintetizada, pois conforme análise do presente estudo, as aplicações possíveis vão além do gerenciamento da ocupação e alocação.

Ainda, compara-se com as definições constantes na literatura, nas quais a gestão de espaço em BIM-FM pode promover soluções, tais quais: a visualização dos espaços e seus atributos, a possibilidade de comparação de situação atual e planejada de forma otimizada, rastreio dos ativos ao longo do tempo [5], regras de medições de espaços automatizadas ou não e, uma intuitiva exibição de plantas que suportam um melhor gerenciamento e uma melhor comunicação em mudanças de cenários [29]. As descrições acima se enquadram com a classe de problemas inventários de espaços. Já as descrições a respeito do acesso imediato que possibilita a identificação de subutilização dos espaços, otimização de agendas dos ambientes e eventos [5], se englobam na classe de problemas ocupação e alocação de espaços.

Constata-se que os autores [5] [29] não fizeram referência aos benefícios que são apresentados em relação ao conforto interno do espaço, simulações de usos e visualização pelo usuário.

Em relação aos agentes envolvidos nos processos, a partir da estratificação da RSL foi possível identificar a preocupação com a visão do usuário do imóvel, presente nas classes de problemas conforto térmico do espaço e visualização do espaço pelo usuário, enquanto as definições de gestão de espaço da literatura abordam exclusivamente a visão de gerentes e proprietários [5] [28] [29].

Ainda, destacam-se as abordagens sobre a extensão do uso do modelo BIM ocasionada por meio da integração com novas tecnologias como loT e o uso de gêmeos digitais para a gestão de espaços.

Apesar da diversidade de tipos de edificações utilizadas nas pesquisas da amostra, as universidades se destacam como indutoras de implementações de novas soluções.

Finalmente, conclui-se que há lacunas nas definições de gestão de espações em BIMFM. Desta forma, em decorrência da dinamicidade no surgimento e desenvolvimento de novas tecnologias e o crescente envolvimento de usuários como agentes nos processos, a proposição de uma nova definição se apresenta como uma oportunidade para trabalhos futuros. 
Ao Programa de Pós-Graduação em Engenharia Civil da Universidade Federal do Paraná (PPGEC-UFPR) e à Universidade Federal do Paraná por proporcionarem a realização deste trabalho.

\section{REFERÊNCIAS}

[1] PUGA, F. P.; CASTRO, L. B. Visão 2035: Brasil, país desenvolvido: agendas setoriais para alcance da meta. 1. ed. Rio de Janeiro: Banco Nacional de Desenvolvimento Econômico e Social, 437 p., 2018. ISBN 9788587545640

[2] EASTMAN, C.; TEICHOLZ, P.; SACKS, R.; LISTON, K. BIM Handbook: a guide to building information modeling for owners, managers, designers, engineers and contractors. 2. ed. Nova lorque: John Wiley \& Sons, 2011.

[3] ASSOCIAÇÃO BRASILEIRA DE NORMAS TÉCNICAS. NBR ISO 41013. Facility management - Escopo, conceitos-chave e benefícios. Associação Brasileira de Normas Técnicas. Rio de Janeiro, 2019.

[4] DRESH, A. Design Science Research: método de pesquisa para avanço da ciência e tecnologia. Porto Alegre: Bookman, 2015. E-pub.

[5] BECERIK-GERBER, B. et al. Application Areas and Data Requirements for BIM-Enabled Facilities Management. Journal of Construction Engineering and Management, v. 138, n.3, p. 431-442, 2012. DOI:10.1061/(ASCE)CO.1943-7862.0000433

[6] LAZAR, S.; MCARTHUR, J. J. BIM for Corporate Real Estate Data Visualization from Disparate Systems. In: EAI INTERNATIONAL SUMMIT, SMART CITY 360, 1, 2015, Bratislava, Toronto. Proceedings [...]. Cham: Springer International Publishing, 2016, p. 504-516.

[7] LATHER, J.; AMOR, R.; MESSNER, J. A Case Study in Data Visualization for Linked Building Information Model and Building Management System Data. In: ASCE INTERNATIONAL WORKSHOP ON COMPUTING IN CIVIL ENGINEERING, 2017, Seattle. Proceedings [...]. Reston: ASCE, 2017, p. 228-235. DOI: https://doi.org/10.1061/9780784480823.028

[8] KIM, E. Field survey system for facility management using BIM model IoT management for facility management. In: INTERNATIONAL CONFERENCE OF THE ASSOCIATION FOR COMPUTER-AIDED ARCHITECTURAL DESIGN RESEARCH IN ASIA, 23., 2018, Hong Kong. Proceedings [...]. Honk Kong: CAADRIA, 2018, p. 535-544.

[9] ILTER, D. et al. Modelling Information Flow of Occupant Feedback in Office Buildings. In: INTERNATIONAL SYMPOSIUM ON AUTOMATION AND ROBOTICS IN CONSTRUCTION, 35., 2018, Berlin. Proceedings [...]. Berlim: ISARC, 2018, p. 811-818. DOI: https://doi.org/10.22260/ISARC2018/0113

[10] HALMETOJA, E. The conditions data model supporting building information models in facility management, Facilities, v. 37, p. 484-501, 2019. DOI: 10.1108/F-11-2017-0112

[11] XIE, X. et al. Visualised inspection system for monitoring environmental anomalies during daily operation and maintenance. Engineering, Construction and Architectural Management, v. 27, n. 8, p. 1835-1852, 2020. DOI: https://doi.org/10.1108/ECAM-112019-0640.

[12] COSTIN, A.; TEIZER, J. Utilizing BIM for Real-Time Visualization and Indoor Localization of Resources. In: INTERNATIONAL CONFERENCE ON COMPUTING IN CIVIL AND BUILDING ENGINEERING, 2014, Orlando. Proceedings [...]. Reston: ASCE, 2014, p. 649-656.

[13] TELES, R. P.; AMORIM, A. L. Alocação de Espaços: Uma aplicação de Facilities Management. In: SIMPÓSIO BRASILEIRO DE TECNOLOGIA DA INFORMAÇÃO E 
COMUNICAÇÃO NA CONSTRUÇÃO, 1; SIMPÓSIO BRASILEIRO DE GESTÃO E ECONOMIA DA CONSTRUÇÃO, 10, 2017, Fortaleza. Anais [...]. Porto Alegre: ANTAC. 2017, p. 151158.

[14] SOLLA, M. et al. Integration of BIM and Archibus for Facility Management (FM) in FKAAS, UTHM Building. In: INTERNATIONAL CONFERENCE ON CIVIL AND ENVIRONMENTAL ENGINEERING FOR SUSTAINABILITY, 5, Johor, 2019. Proceedings [...]. Bristol: IOP Publishing Ltd., v.498, 2020, n. 012088.

[15] MORETTI, N. et al. Maintenance service optimization in smart buildings through ultrasonic sensors network. Intelligent Buildings International, v.13:1, p. 4-16, 2021. DOI: https://doi.org/10.1080/17508975.2020.1765723

[16] TAMKE, M. et al. From Point Clouds to Definitions of Architectural Space : Potentials of Automated Extraction of Semantic Information from Point Clouds for the Building Profession. In: INTERNATIONAL CONFERENCE ON EDUCATION AND RESEARCH IN COMPUTER AIDED ARCHITECTURAL DESIGN IN EUROPE, 32, 2014, Newcastle. Proceedings [...]. Brussel: eCAADe, v.2, 2014, p. 557-566.

[17] MCGINLEY, T.; FONG, D. DesignGhosts: Mapping occupant behaviour in BIM. In: INTERNATIONAL CONFERENCE OF THE ASSOCIATION FOR COMPUTER-AIDED ARCHITECTURAL DESIGN RESEARCH IN ASIA, 20, 2015, Hong Kong. Proceedings [...]. Hong Kong: CAADRIA, 2015, p. 365-374.

[18] RESENDE, R. et al. Plataforma Web-BIM para Gestão de Instalações de um Campus. In: CONGRESSO PORTUGUÊS DE BUILDING INFORMATION MODELLING, 1, 2016, Guimarães. Proceedings [...]. Lisboa: ISCTE, 2016, p. 501-512.

[19] ATAZADEH, B. et al. Extending a BIM-based data model to support 3D digital management of complex ownership spaces. International Journal of Geographical Information Science, v. 31, p. 499-522, 2016. DOI: http://dx.doi.org/10.1080/13658816.2016.1207775

[20] ATAZADEH, B. et al. Utilizing a Building Information Modelling Environment to Communicate the Legal Ownership of Internet of Things-Generated Data in Multi-Owned Buildings. Electronics, v. 8, 2019. DOI: http://dx.doi.org/10.3390/electronics 8111258

[21] SUTER, G. Modeling multi-level spaces with network-based space layouts: a case study. In: WORKSHOP OF THE EUROPEAN GROUP FOR INTELLIGENT COMPUTING IN ENGINEERING, 21, 2014, Cardiff. Proceedings [...]. Berlin: EG-ICE, 2014.

[22] BAKOWSKI, J. Analytical Tools for Functional Assessment of Architectural Layouts. In: WORLD MULTIDISCIPLINARY CIVIL ENGINEERING-ARCHITECTURE-URBAN PLANNING SYMPOSIUM, Prague, 2017. Proceedings [...]. Bristol: IOP Publishing Ltd, v. 245, 2017, n. 042044.

[23] VENTURA, S.M et al. Evaluation of Building Use Scenarios by Crowd Simulations and Immersive Virtual Environments: A Case Study. In: INTERNATIONAL SYMPOSIUM ON AUTOMATION AND ROBOTICS IN CONSTRUCTION, 35, 2019, Berlin. Proceedings [...]. Berlim: ISARC, 2018, p. 554-561.

[24] JI, S. Y.; KIM, M.; JUN, H. Space Management on Campus of a Mobile BIM-based Augmented Reality System. Architectural research, v. 19, p. 1-6, 2017. DOI: https://doi.org/10.5659/AIKAR.2017.19.1.1

[25] LATHER, J.; LEICHT, R.; MESSNER, J. Engaging with BIM: Interactive Workspaces in Facility Design and Construction. In: CONSTRUCTION RESEARCH CONGRESS, 2018, New Orleans. Proceedings [...]. Reston: ASCE, 2018, p. 765-775.

[26] PISPIDIKIS, I. et.al. Combining methodological tools for the optimum 3D modelling of ntua campus. In: 3D Geolnfo Conference, 2018, , Delft. Proceedings [...]. Hannover: ISPRS, 2018, p. 57-63. 
[27] CHIAS, P. et al. 3D modelling and virtual reality applied to complex architectures: An Application to Hospitals' Design. In: INTL. WORKSHOP 3D-ARCH, 2019, Bergamo. Proceedings[...]. Hannover: ISPRS, 2019, p. 255-260.

[28] BIM DICTIONARY. Space Management, version 1.2019. Disponível em: https://bimdictionary.com/en/space-management/1. Acesso em: 1 fevereiro 2021.

[29] TEICHOLZ, P. BIM for Facility managers. 1. Ed. Nova lorque: John Wiley \& Sons, 2013. 